\title{
Réécrire le passé pour angoisser le présent
}

Essai sur la « nouvelle histoire » catalane

Reescribir el pasado para sembrar zozobra en el presente

\section{Mathias Ledroit}

\section{(2) OpenEdition}

\section{Journals}

Édition électronique

URL : https://journals.openedition.org/agedor/5619

DOI : 10.4000/agedor.5619

ISSN : 2104-3353

Éditeur

Laboratoire LISAA

Référence électronique

Mathias Ledroit, « Réécrire le passé pour angoisser le présent », L'Âge d'or [En ligne], 13 | 2020, mis en ligne le 01 octobre 2021, consulté le 14 octobre 2021. URL : http://journals.openedition.org/agedor/ 5619 ; DOI : https://doi.org/10.4000/agedor.5619

Ce document a été généré automatiquement le 14 octobre 2021.

L'Âge d'or. Images dans le monde ibérique et ibéricoaméricain 


\title{
Réécrire le passé pour angoisser le présent
}

\author{
Essai sur la « nouvelle histoire » catalane \\ Reescribir el pasado para sembrar zozobra en el presente
}

\section{Mathias Ledroit}

\author{
L'histoire est un appel au calme ${ }^{1}$ \\ El complejo colectivo de víctimas \\ suele crear otros verdugos ${ }^{2}$.
}

1 La "Nova Història"3, la «nouvelle histoire ", est un courant de pseudo-histoire qui apparaît en 2007, sous l'impulsion de Jordi Bilbeny et d'Albert Codinas ${ }^{4}$. Elle se propose d'entreprendre des recherches sur "la tergiversació de la història que Catalunya [...] va patir i pateix encara, per part de la corona castellana, corona que va acabar apropiantse del poder de la monarquia hispànica" ${ }^{5}$. La diffusion de ces thèses est assurée principalement par deux sites internet : l'Institut Nova Història (dorénavant INH), mis en ligne en 2007, et le Cercle Català d'Història (dorénavant $\mathrm{CCH}$ ), lancé en $2008^{6}$. Ce dernier se présente comme une association dont le propos est de «promoure la recuperació de la memòria històrica mitjançant el coneixement de la història de Catalunya, via l'anàlisi, recerca, debat i divulgació de la nostre història $[. ..] »^{7}$.

2 Ces deux sites ont fait l'objet de vives critiques, notamment de la part d'historiens professionnels qui leur ont reproché de diffuser des théories pittoresques qui, selon Àngel Casals par exemple, historien moderniste de l'Université de Barcelone, discréditent la Catalogne et le travail des universitaires ${ }^{8}$. Aussi serait-on tenté, a priori, de simplement passer son chemin et de ne pas s'arrêter sur ces théories. Que dire, en effet, à des personnes capables de revendiquer la catalanité de Christophe Colomb, de Léonard de Vinci et de Teresa de Jesús, ou encore de dire que Cervantès a usurpé l'autorité du Quichotte à un écrivain catalan? Comment réagir face à des théories qui cherchent à prouver que le drapeau des États-Unis ou le maillot des joueurs du R.C. Lens sont tout bonnement des copies de la senyera ${ }^{9}$ ? Or, force est de constater que ces thèses sont tout aussi vivement critiquées qu'elles sont amplement applaudies, dans les cercles nationalistes et indépendantistes, mais aussi en place publique, puisqu'elles ont 
reçu le soutien officiel de certaines organisations politiques ou de municipalités. En 2013, par exemple, l'INH a reçu le XIX ${ }^{\mathrm{e}}$ prix national President Lluís Companys d'Esquerra Republicana de Catalunya, pour "su labor de investigación, estudio y divulgación de la historia de Cataluña, con un carácter iconoclasta e innovador, explotando al máximo las posibilidades de interacción, participación e internacionalización de los estudios, y por su compromiso con el conocimiento de la historia de Cataluña" ${ }^{10}$.

3 Certains spécialistes, à l'instar d'Henry Kamen, ont cherché à mettre en lumière la manipulation historique qui préside dans l'historiographie indépendantiste, notamment celle sur laquelle s'appuient les chefs-de-file du "procés". Dans un essai paru en 2014, à l'occasion des commémorations du Tricentenaire de 1714, Henry Kamen avait cherché à mettre un terme à ce qu'il appelait "la escalada de desinformación que se está perpetrando por todas partes"11, considérant que "cada país tiene el derecho a exagerar sus propios éxitos [...]. La tarea del historiador es presentar documentación para explicar que aquello no fue así"12. Il argumentait, en effet, que

Es una lástima que la historia de Cataluña haya sido inadecuadamente estudiada por los historiadores, y sistemáticamente distorsionada por ideólogos, políticos y periodistas que, con mucha frecuencia, basan sus discursos en información poco fiable. A lo largo de su historia, Cataluña ha sido víctima de ciertos procesos de desinformación fomentados por aquellos que prefieren no esforzarse en el estudio del pasado. ${ }^{13}$

4 C'est là, semble-t-il, une entreprise vaine, car, au fond, ce qui anime ces «nouveaux historiens » n'est ni l'objectivité ni la rationalité, ni même la rigueur scientifique et ce, malgré les déclarations d'intention qu'on trouve çà et là dans les différents articles et par lesquelles ils rappellent, pour se donner une certaine crédibilité, que l'histoire est une science et qu'en tant que telle, elle repose sur une exigence de qualité et de rigueur ${ }^{14}$. En réalité, plus les historiens s'efforcent d'armer un contre-discours destiné à « rétablir la vérité », plus ils consolident, malgré eux, les thèses de cette nouvelle histoire, dont la force réside dans le fait que la rhétorique et les émotions aient pris le dessus sur la démarche et la preuve scientifiques, ainsi que sur le document d'archive. D'ailleurs le simple fait d'émettre une critique permet à ces « nouveaux historiens » de dire qu'ils ont mis le doigt sur un point sensible et les conforte dans leur certitude que l'histoire officielle est manipulée.

5 Pour aussi « pittoresques » qu'elles soient, ces théories doivent cependant retenir une partie de notre attention, non pas pour les contester mais davantage pour tenter de comprendre les rouages de cette rhétorique et, d'autre part, pour tâcher de discerner ce qu'elles ont à nous dire sur la société qui les produit et, simultanément, les consomme. Il ne sera pas question, dans cet essai, de s'interroger sur la pertinence ni même de tenter de mettre en lumière le caractère absurde de ces élucubrations, et encore moins de les dénoncer. Nous nous proposons d'envisager cette «nouvelle histoire » comme un système doté d'une cohérence interne - qu'on pourra, à juste titre, considérer comme incohérent, peu importe, la question n'est pas là. En effet, tout système de représentation ${ }^{15}$, aussi loufoque soit-il, repose sur un ensemble de structures et sur une dynamique articulée dont il s'agira de proposer un premier essai de synthèse.

6 Ce qui relie ces deux sites, c'est l'urgence de dire la violence et l'oppression que l'État espagnol, et en particulier la Castille, exerce sur la Catalogne. Jordi Bilbeny déclare en effet que "L'Estat xucla la ment, l'absorbeix i [els seus] historiadors defensen la versió oficial, la dels que paguen. Això fa que quedi una pseudohistòria i creen la realitat com 
volen." ${ }^{16}$ Dominique Fisher a défini l'écriture de l'urgence comme étant «motivée et générée par une situation extrême ", ici, en l'occurrence, les répressions supposément exercées par l'Espagne sur la Catalogne, depuis plusieurs siècles.

\section{Le concept de censure}

7 Le nouveau modèle historiographique prôné par l'INH et par le $\mathrm{CCH}$ repose sur le principe recteur de la « censure ", introduit par Jordi Bilbeny, véritable mentor de cette «nouvelle histoire », que certains n'hésitent pas à qualifier d'«Indiana Jones » de la Catalogne, "un català que des de la fascinació refà el trajecte que ens ha portat als nostres dies seguint rastres i senyals que han estat ocultats i enterrats sota una gruixuda capa de pols "17. Jordi Bilbeny est un historien auto-proclamé : licencié en philologie catalane de l'Universitat Autònoma de Barcelona, il a entrepris une thèse en histoire à l'Universitat de Barcelona sur la censure des textes bibliques exercée par l'Église. Parallèlement, il a engagé des recherches pour écrire un roman historique sur Christophe Colomb, au cours desquelles il aurait percé à jour les manipulations auxquelles se serait livré l'État espagnol, grâce à la censure, pour s'approprier la découverte de l'Amérique. Dans un ouvrage intitulé, de façon très suggestive, Brevíssima destrucció de la història, publié en 2014 chez Librooks, il fait toute la lumière sur cette manipulation et entreprend de rétablir la "vérité » sur les expéditions maritimes catalanes vers l'Amérique ${ }^{18}$ : Christophe Colomb ne serait pas génois mais catalan; il aurait été financé par des banquiers catalans et sa première expédition ne serait pas partie de Palos de la Frontera en Andalousie mais de Pals, en Catalogne. Ces recherches, qu'il dit mener depuis vingt-cinq ans, ont donné lieu à plusieurs ouvrages, à de nombreux articles et conférences dont l'objectif avoué est de lever le voile sur la façon dont "treballa la maquinària censora" et dont "ens han rentat el cervell"19. Selon lui, depuis le $\mathrm{XvI}^{\mathrm{e}}$ siècle, l'histoire de la Catalogne a été altérée par la Castille, celle-ci ayant cherché, par l'intermédiaire de la censure, de la manipulation, de l'occultation, de l'appropriation et de la falsification, à assurer son leadership idéologique dans la construction d'un État espagnol centralisateur. La généalogie de cette censure est variable selon les articles et selon les auteurs. Si certains la font remonter à l'union dynastique de 1469 entre Isabel de Castille et Ferdinand d'Aragon, d'autres la situent au Compromis de Caspe en 1412, ou en 1714, avec le décret de Nueva Planta. D'autres encore la situent en 1875, au moment de la Restauration des Bourbons.

La méthode consiste donc à partir du principe que les institutions étatiques, prises en otage par les Castillans, ont exercé une censure sur les publications et se sont livrées à une réécriture du discours historique. Le $\mathrm{CCH}$ et l'INH entendent donc "revisar la Història que ens han inculcat fins ara" et proposer un nouveau modèle historiographique dépourvu des effets de cette censure, encore pratiquée aujourd'hui, pour diffuser une version qui ne soit pas «interessada, amb retallades, personatges desapareguts, autors traduïts l'obra original dels quals ha desaparegut $»^{20}$. Leur démarche consiste à jouer sur ce qu'on appelle en historiographie les «effets de source ", autrement dit les lacunes documentaires ou encore les incohérences que la documentation peut présenter. Ces vides laissés par la documentation sont interprétés comme des manipulations orchestrées par les censeurs dans le but dissimulé de créer une distorsion de l'histoire qui agirait à leur profit. De là, la nécessité d'entreprendre une révision historiographique, de proposer une «nouvelle histoire » placée sous l'ère 
du soupçon pour mettre en lumière les incohérences de l'histoire officielle, les violences infligées par l'État aux Catalans, tout en l'inscrivant dans la longue durée. Il y aurait, en effet, d'une part, la censure exercée par la Castille dans le processus de création de l'État moderne depuis l'époque des Rois Catholiques. Mais l'union dynastique de 1469 aurait également donné lieu à une révision de l'histoire médiévale dans le but de gommer progressivement, par l'intermédiaire de la censure, l'histoire de la Catalogne de la mémoire collective pour dissimuler l'annexion de la Principauté et pour assurer la soumission des Catalans : "La investigació i la divulgació de la nostra història juguen el paper de desfer les mentides difoses sistemàticament i reiterada des del Ministerio de la Verdad [...] de l'Estat Español, borbònic o no, que és per a nosaltres l'encarnació del Gran Germà definit a 1984." ${ }^{21}$ La référence au ministère de la Vérité Miniver en novlangue - suggère que l'histoire de la Catalogne, telle que nous la connaissons aujourd'hui, n'est que le résultat d'une vaste entreprise de propagande menée par l'État espagnol afin d'assurer sa domination: "Cal mirar al passat per no oblidar els mèrits i el valor de tots aquells que el van configurar i hem de veure amb absoluta claredat que l'oblit i el desenteniment generen ignorància i que aquesta és el millor camp de cultiu per continuar perpetuant la manipulació i la dominació." ${ }^{22}$ Privés de leurs droits démocratiques, les Catalans auraient été soumis à un long processus de "déculturation ». Dès lors se pose la question de savoir comment l'État espagnol a exercé cette censure ${ }^{23}$. Chronologiquement, l'union des Couronnes de Castille et d'Aragon coïncide avec le début de ce que les écrivains de la Renaissance catalane du $\mathrm{XIX}^{\mathrm{e}}$ siècle ont appelé «Décadence ». Cette thèse, encore fort présente dans les esprits bien qu'on en ait montré les limites, est impulsée, entre autres, par Valentí Almirall Llotzer dans son essai intitulé Lo Catalanisme, paru en 1886. Pour faire court, l'auteur y défend la thèse selon laquelle l'union dynastique des Rois Catholiques en 1469 et la découverte de l'Amérique en 1492 auraient été à l'origine de l'entrée de la Catalogne dans une période de décadence à la fois politique, économique et culturelle, ce que les intellectuels de la Renaixença auraient œuvré à corriger. Jordi Bilbeny, dans un ouvrage paru en 2014 sous le titre de Decadència i Inquisició, s'interroge sur cette décadence de la littérature en langue catalane. La question, en soi, est légitime et pose de nombreuses difficultés interprétatives. Car comment expliquer la soudaineté du passage entre une littérature qui connaît son âge d'or au $\mathrm{Xv}^{\mathrm{e}}$ siècle, et son apogée avec le poète valencien Ausiàs March, à une littérature quantitativement moindre, phénomène qui s'étend de l'extrême fin $\mathrm{du} \mathrm{Xv}^{\mathrm{e}}$ siècle jusqu'au xIx ${ }^{\mathrm{e}}$ ? Le raisonnement de Bilbeny s'appuie sur des conjectures et cherche à relever une série de coïncidences. La Décadence de la littérature catalane est non seulement contemporaine de l'union dynastique, qui marque les premiers temps de la construction de l'État moderne, mais également de l'instauration de l'Inquisition moderne, en 1478 dans les territoires castillans et de son implantation en 1483 dans ceux de la Couronne d'Aragon. Elle coïncide également avec l'introduction de l'imprimerie et de la promulgation du décret royal de 1502, par lequel les Rois Catholiques généralisent la censure préalable à toute publication dans les deux couronnes. Institutionnalisée, la censure aurait eu deux conséquences majeures : d'une part, les auteurs de langue catalane, à l'instar de Juan Boscán, Antoni Beuter, Lluís Ponç Icart et Jeroni Pujades, auraient été contraints de traduire leurs œuvres en castillan : “[...] els autors catalans van ser forçats, mitjançant coaccions i amenaces a traduir al castellà les seves obres. Un canvi que històricament s'ha intentat explicar com un procés natural, però que implica un veritable genocidi lingüístic i cultural." ${ }^{24} \mathrm{~A}$ l'inverse, ceux qui, comme Cristòfor Despuig, auteur des Colloquis de la insigne ciutat de 
Tortosa, qu'il a écrits en catalan, auraient été condamnés à voir leurs œuvres rester au stade du manuscrit jusqu'au $\mathrm{XIX}^{\mathrm{e}}$ siècle ${ }^{25}$. D'autre part, la censure inquisitoriale aurait donné lieu à un pillage culturel : des œuvres initialement écrites en catalan auraient été usurpées par des Castillans qui les auraient traduites et se les seraient appropriées :

La Decadència ha estat això: la usurpació total, absoluta, abassegadora i premeditada de la cultura catalana i bona part del XVII per part d'una nova estructura emergent: l'estat espanyol, amb disseny castellà. Començant per en Colom i acabant amb el Servent, per dir-ho de forma resumida i cenyint-me a dos dels pilars més gruixuts, més irradiadors i més universals. ${ }^{26}$

Joan Miquel Servent, dont il est question dans la citation, est, avec Christophe Colomb, l'un des deux personnages saillants de l'histoire catalane que la Castille aurait usurpés à la Catalogne. Il s'agirait du véritable auteur du Quichotte, que Miguel de Cervantès aurait spolié puis traduit en castillan pour se l'attribuer et pour en faire l'œuvre littéraire de référence du Siècle d'Or espagnol. En réalité, cette thèse varie selon les auteurs. Pour Lluís Maria Mandado, auteur d'un ouvrage intitulé El Quixote va esborrar el Quixot ${ }^{27}$, Cervantès aurait tout simplement usurpé l'œuvre initialement écrite en catalan et l'aurait traduite en castillan, conformément aux injonctions de l'appareil de censure de l'État espagnol. Jordi Bilbeny, lui, estime que la censure s'est exercée sur Joan Miquel Servent, originaire d'Alicante, qui aurait écrit le Quichotte. L'État espagnol s'en serait saisi, l'aurait traduit en castillan et aurait également usurpé l'identité de l'auteur pour créer de toutes pièces Miguel de Cervantès. Selon Jordi Bilbeny, la seconde partie du Quichotte, publiée en 1615, contient de nombreux indices qui accréditent cette thèse. Il écrit, en effet, être interpellé par les descriptions précises des galères dans le port de Barcelone et en conclut que Cervantès n'a rien inventé et qu'il s'est contenté de les décrire depuis la fenêtre de la maison qu'il possédait sur l'actuel passeig Colom ${ }^{28}$. De même, il mentionne le personnage de Rocaguinarda, inspiré du bandoulier Perot Rocaguinarda (1582-1635), contemporain de Cervantès, qui, par son mode de vie " terroriste ", était un dissident et, par conséquent, ne pouvait être admiré que par un Catalan lui-même opprimé par l'État espagnol :

\footnotetext{
El bandoler Perot de Rocaguinarda. Amb noms i cognoms, i li dedica els elogis més sincers del Quixot! En aquell moment, no era un personatge de novella: en aquella època el seu cap tenia un preu, atemptava contra les estructures (i les persones!) que representaven la monarquia... o sigui, que era un terrorista de l'època. Per tant, Cervantes què fa? Defensa el rei o els que lluiten contra ell? El Quixot és boig, però Cervantes no: utilitza uns mecanismes per atacar uns personatges i lloar-ne uns altres. $^{29}$
}

10 On pourrait également citer - au risque de multiplier les exemples - le cas du Lazarillo de Tormes $^{30}$. Sans entrer dans les détails de la démonstration ${ }^{31}$, l'introduction de La vida de Llàtzer Tormos, paru en 2013 aux éditions Llibres de l'Índex - une maison d'édition au nom lui aussi très suggestif -, entend démontrer que l'œuvre de référence de la littérature picaresque a été originalement écrite en catalan par Joan Timoneda ou encore par Joan Lluís Vives. Le jeune protagoniste de l'histoire, Llàtzer, ne serait pas né sur les rives du fleuve Tormes à Tolède mais sur celles du fleuve Tormos, dans la Huerta de Valence. L'intrigue, quant à elle, ne se déroulerait pas à Salamanque mais entre Valence et Gandia. Les éditions de 1554 sont, selon Jordi Bilbeny, encore, des éditions d'État, qui proviennent d'un manuscrit valencien, écrit en catalan, et qui sont adaptées pour que la trame se déroule en Castille et pour gommer les traces de l'apologie que l'œuvre, dans son état originel, fait de la révolte des Germanies de Valence. Aussi faudrait-il en conclure, avec cette nouvelle édition, qu'“una part substancial del Siglo 
de Oro de la llengua castellana podria ser una apropiació en bloc d'autors catalans (de Joan Timoneda a Miguel de Cervantes o de la Celestina a La Vida de Lazarillo de Tormes)" ${ }^{32}$.

Dans ce système, le concept de «censure » est opérant dès lors qu'on part du principe que l'histoire est écrite par les vainqueurs. Étant donné que la Catalogne est la grande perdante de l'Histoire - avec un $\mathrm{H}$ majuscule -, c'est l'Espagne, ou plus précisément la Castille qui a écrit l'histoire de la Catalogne. Aussi cette «nouvelle histoire » est-elle envisagée comme un outil social et politique mis au service d'une vérité et d'une liberté dont les historiens de métier ne peuvent bénéficier, puisqu'ils ont été formés et évoluent dans un système universitaire entièrement aux mains de l'Université espagnole, tant pour ce qui relève des places d'enseignants-chercheurs que des financements des projets de recherche ${ }^{33}$.

Les centres d'archives mêmes deviennent suspects, car ils sont placés sous la tutelle de l'État, lequel, à en croire certains, a appliqué et applique encore une politique de purge documentaire, ce qui explique les lacunes de la documentation ${ }^{34}$. En d'autres termes, les Catalans ont été dépossédés de leur histoire et, partant, victimes d'une injustice : "La història l'han escrit els vencedors [...]. La interpretació que li han volgut donar aquells vencedors i aquelles plomes que han anat narrant històries del nostre passat, les quals no sempre han reflectit amb justícia els fets esdevinguts." ${ }^{35}$ Ce qui a été enseigné, notamment dans les livres et les écoles, ne serait donc pas la version fidèle de la réalité mais bien une version manipulée: "Molt sovint, aquest coneixement del que érem realment com a catalans i com a país o nació no és conegut per les generacions actuals, perquè mai s'ha ensenyat a les escoles, perquè sempre s'ha volgut ocultar per part d'aquells que no els interessa que es conegui." ${ }^{36}$ Aussi cette "nouvelle histoire " propose-t-elle une démarche heuristique qui ne repose pas sur une analyse ou une lecture du passé, ni même sur une expérience d'« estrangement », mais qui consiste en une réaction militante contre l'entreprise de manipulation à laquelle l'Espagne s'est livrée pour assurer sa domination. Dit encore autrement, l'histoire officielle est le résultat d'une usurpation, voire d'une spoliation de l'histoire catalane par l'État espagnol, lequel est jugé coupable d'« historicide » et de génocide culturel.

\section{Spoliation, « historicide » et génocide culturel}

13 À l'inverse de la censure, qu'on avait définie comme un principe recteur, la spoliation est une théorie explicative destinée, en premier lieu, à trouver une justification rationnelle face à la raréfaction de la documentation en langue catalane à l'époque moderne, entre la fin $\mathrm{du} \mathrm{xv}^{\mathrm{e}}$ siècle et la Renaixença de la seconde moitié du $\mathrm{xIX}^{\mathrm{e}}$. L'enquête ainsi menée se cristallise, au sens presque stendhalien du terme, autour de trois faits historiques : la prétendue persécution de la langue catalane, dès l'avènement des Rois Catholiques ${ }^{37}$, la disparition brutale de la littérature catalane à cette même époque $^{38}$ - fortement liée au point précédent - et, plus important encore, la catalanité occultée de Christophe Colomb ${ }^{39}$. Cette spoliation historique, ou " historicide », est une entreprise destinée à effacer méthodiquement les traces de l'histoire catalane de la mémoire collective, dans le but d'assimiler la Catalogne et d'encourager chez les Catalans une mauvaise estime de soi, pour favoriser leur docilitée ${ }^{40}$ :

Tot això es va fer per tal que l'apropiació d'aquests territoris catalans i les seves riqueses no es digués en el futur que havia estat un robatori [...]. Nosaltres ja sabem 
que els espanyols, si es tracta de robar i espoliar, són uns mestres, i pel que fa a canviar la història el mateix. ${ }^{41}$. discours historique. La spoliation historique de la découverte de l'Amérique, c'est aussi et avant tout la spoliation de l'or américain qui aurait dû revenir aux Catalans et dont les Castillans se sont emparés. On perçoit ici un parallèle avec le slogan véhiculé par la gauche républicaine catalane "Espanya ens roba". C'est aussi une réactivation d'un discours aujourd'hui effacé dans le discours officiel de l'indépendantisme de la rivalité entre Catalans et Andalous, les premiers payant pour les seconds. L'Andalousie, d'où partaient toutes les expéditions jusqu'à la levée du monopole au XVIII siècle, a spolié l'or américain qui aurait dû revenir aux Catalans, tout comme elle profite, aujourd'hui, des impôts des Catalans conformément au système de rétribution en vigueur. c'est davantage la démarche heuristique et le vocabulaire employé. En effet, dans un article intitulé "Mètode historiogràfic o policial? Reflexions d'una espoliació historiogràfica" ${ }^{42}$, Joaquim Ullan Martorell déclare, dans les premières lignes, que l'historiographie est une science qu'il rapproche volontiers de la mathématique. Le travail de l'historien doit dès lors être rigoureux, dépourvu d'a priori et doit se fonder sur des documents historiques. Le cas de la Catalogne est toutefois particulier, on l'a vu, car non seulement la documentation est lacunaire voire inexistante, mais aussi parce que celle qui nous est parvenue a fait l'objet d'une censure institutionnelle par l'État espagnol depuis cinq-cents ans. En d'autres termes, la disparition ou l'altération des principaux documents originaux, comme le journal de Christophe Colomb par exemple, ne sont pas dues aux vicissitudes du temps ni même aux modes de conservation des archives, mais résulte d'une destruction programmée par l'État et l'Inquisition. Aussi n'est-il pas possible de se fier à la documentation officielle: "El estado español persiguió, censuró y secuestró la mayor parte de los escritos que trataban del descubrimiento y la conquista [...]. Se prohibieron o se secuestraron las obras escritas por los propios conquistadores y frailes protagonistas de la conquista." ${ }^{43}$ Andreu Mas, dans un article publié sur le site de l'INH, avance quant à lui que "Espanya mai reconeixerà que la descoberta d'Amèrica és catalana, perquè això trencaria una gran mentida històrica que sustenta el mite de l'imperi espanyol [...] tot plegat forma part d'un procés d'espoliació cultural molt lligat al d'espoliació econcòmica". Et d'ajouter que "Potser d'aquí a uns anys diran que El Bulli era a Almeria." ${ }^{44}$

Il incombe par conséquent à l'historien de recourir à d'autres méthodes et notamment à une méthode "autènticament policial" pour reconstituer le passé grâce aux indices que le temps a, pour ainsi dire, laissés sur la scène de crime. Ce n'est toutefois pas la méthode indiciaire, théorisée par Carlo Ginzburg et la micro-histoire ${ }^{45}$, qui est mise en œuvre. Sans le formuler explicitement, Joaquim Ullan Martorell conçoit plutôt l'enquête comme un puzzle et s'inspire de la méthode déductive et de mise en corrélation des indices de Sherlock Holmes ${ }^{46}$, comme le montrent ses "Cróniques des de la Sevilla colombina" ${ }^{47}$. Mais plus important encore, cette méthode policière comporte une forte charge symbolique. Car le but de l'enquête n'est pas de reconstituer le passé mais bien d'instruire un dossier à charge contre l'Espagne, en prouvant sa culpabilité dans la manipulation des faits et des archives, et, simultanément, contre les "col-laboradors de l'acusat"48, c'est-à-dire tous ceux qui mettraient des «bâtons dans les roues $»^{49}$ des enquêteurs du CCH. Le terme "colllaboradors" n'est pas neutre et n'est pas 
une simple commodité lexicale pour désigner l'obstruction à la justice. Il s'agit bien d'un réemploi du lexique associé à la Seconde Guerre Mondiale.

On retrouve une démarche similaire dans un ouvrage publié en 2015 par Antoni Muñoz et Josep Catà, du $\mathrm{CCH}$, sur la légation des ambassadeurs de la Generalitat à Madrid en 1640 , dans la période qui précède l'éclatement de la grande révolte des Catalans ${ }^{50}$. Antoni Muñoz et Josep Catà replacent cette mission diplomatique dans le contexte des soulèvements populaires survenus en Catalogne contre les soldats des tercios. Ils entendent ainsi mettre en lumière la façon dont la révolte populaire (mieux connue dans l'historiographie sous le nom de "Guerra dels Segadors") s'est progressivement politisée au point d'entraîner, en janvier 1641, la proclamation de la république catalane puis l'acclamation de Louis XIII comme Comte de Barcelone. D'un point de vue méthodologique, l'approche des auteurs est ouvertement tendancieuse. Leur but est, en effet, de souligner l'oppression dont la Catalogne a été victime en 1640-1641. Ils s'inscrivent ainsi dans le discours victimisant qui caractérise l'essentiel de l'historiographie catalane des vingt dernières années. L'ouvrage se situe dans le domaine de la mémoire et, serions-nous tentés de dire même, à cheval entre la "mémoire manipulée " et la "mémoire obligée » pour reprendre les termes de l'analyse de Paul Ricœur ${ }^{51}$. L'ouvrage offre une narration argumentée et linéaire de la montée des tensions entre le gouvernement royal et les institutions catalanes jusqu'à la rupture de 1641. Ils choisissent délibérément de faire porter leurs efforts sur la politique vexatoire d'olivares plutôt que sur les tentatives d'apaisement auxquelles ont pourtant participé, certes en vain, les ambassadeurs de la Generalitat ${ }^{52}$. Leur propos consiste à se livrer à une lecture "alternative", selon leur propre terme, de l'enchaînement des événements, en procédant à une sélection de documents d'archives et de citations pour confronter le point de vue des victimes à celui des vainqueurs - ou plutôt des oppresseurs selon leur " point de vue »- qu'avaient restitué des historiens comme John H. Elliott, Josep Sanabre ou encore Eulogio Zudaire. La Catalogne et les " patriotes » se trouvent ainsi installés, au sens canonique du terme, en victimes de la catalanophobie madrilène.

Le choix du mode narratif qu'opèrent les auteurs plonge le lecteur dans une succession linéaire de décisions « cyniques » du Comte-Duc et de ses ministres qui éprouvaient une " authentique délectation » à l'idée d'envahir militairement la Catalogne. Les excès des soldats sont donc décrits comme le déploiement d'une politique programmée de répression et d'une "solution finale». Il est renforcé par tout un système d'adjectivation - "pauvres» Catalans, «une décision profondément injuste» - par lequel les auteurs expriment leur "point de vue", autrement dit, par lequel ils se posent en juges de l'histoire. Ce qui confortera le lecteur dans sa conviction que la Castille, totalitaire, centralisatrice et uniformisatrice, a envahi puis occupé la Catalogne à partir de 1640, opération qu'elle parachève durablement à partir de 1714 .

\section{Le complexe de la sortie d'Égypte}

19 La date de 1714 et le décret de Nueva Planta de 1716 marquent la perte de l'État catalan, le démantèlement de la nation catalane et inaugurent une période d'occupation exercée par une dynastie étrangère, celle des Bourbons, qui applique à la Catalogne une politique colonialiste identique à celle imposée à l'Amérique, l'Asie et l'Afrique. Les 
enquêtes menées s'attachent à recueillir des indices de cette occupation, en les présentant souvent sous forme de listes :

- l'abolition des fueros et des institutions locales, véritables symboles de la « liberté » dont les Catalans jouissaient avant l'occupation ;

- le transfert de l'université de Barcelone à Cervera, ville qui a pris le parti des Bourbons dans la Guerre de Succession;

- l'occupation militaire, mise en relation avec l'occupation nazi de l'Europe dans les années 1940 : "Per començar, els exèrcits de la metròpoli ocupen sense cap dret un país que fins aleshores era sobirà. Catalunya va ser ocupada pels exèrcits de Castella entre 1705 i 1714" ${ }^{33}$;

- l'imposition de la langue castillane dans les institutions et prohibition de la langue catalane, cette dernière étant volontairement confinée dans la sphère privée afin qu'elle disparaisse progressivement : "I per reblar la clau es va posar en pràctica una formidable maniobra de desnacionalització [...]. La llengua catalana va ser prohibida en l'àmbit polític, judicial i acadèmic. Es desfermaria una brutal persecució [...]. El propòsit era [...] reduir el català a l'esfera de la gent rústica i illetrada i provocar-ne la mort" ${ }^{24}$;

- l'instauration de l'absolutisme, dont la définition ressemble davantage à celle de la tyrannie, voire à la dictature qu'à la doctrine définie par Jean Bodin : "Dit això, és fàcil entendre que el desballestament de l'edifici polític català, articulat en el Decret de Nova Planta, aplegava un caràcter punitiu - de càstig -, una naturalesa castellanista -de conquesta - $\mathrm{i}$ una voluntat absolutista - de sistema polític. S'ha dit que Felip $\mathbf{V}$ sentia un odi irracional cap als catalans. És cert, la documentació coetània ho posa de relleu. [...] el desballestament de les institucions catalanes $i$, sobretot, la seva substitució per un model polític i administratiu de fàbrica castellana obeïen, principalment, una qüestió política. El model castellà a diferència del català, era bàsicament vertical. Acusadament jerarquitzat. Era, en definitiva, una plataforma idònia per a la implantació del règim absolutista" ${ }^{25}$;

- la promotion d'organisations qui agissent comme agents de l'intérieur, ce qui introduit, dans le champ politique, la notion d'ennemi de l'intérieur dont il faudrait désormais purger la Catalogne, une thèse qui apparait en pseudo-histoire à partir de 2015 : "per donar soport a la potència ocupant $\mathrm{i}$ frenar els esmentats moviments d'alliberament" ${ }^{56}$. Certains articles ou certains ouvrages, moins nombreux du reste, s'intéressent également à ceux qui sont considérés comme les ennemis intérieurs ${ }^{57}$, ces Catalans qui "van colllaborar en l'ocupació militar i la institucionalització del nou règim" ${ }^{58}$;

- la mise en place, à partir de 1715, d'une nouvelle politique fiscale destinée à organiser et institutionnaliser la spoliation économique de la Catalogne: "[...] la potència ocupant organitza l'espoli econòmic. Recordem el drenatge de l'or americà cap a Castella després de la conquesta. [...] Des de l'impost borbònic del cadastre al segle XVIII fins als setze milions anuals de defècit fiscal dels darrers temps, Catalunya ha estat, durant tres-cents anys, un país espoliat per Espanya" ${ }^{59}$;

- et enfin, exil des partisans de l'archiduc Charles, que certains comparent à la Retirada. En effet, pour certains, la Guerre Civile, à l'instar de la Guerre de Succession, ne serait pas des guerres civiles mais des guerres organisées par l'État contre la Catalogne : "El paralllelisme és sorprenent i aterridor: execucions sense garanties jurídiques, exili massiu, incautacions de béns, espoliació fiscal, obsessió per l'ordre públic, pànic i delacions, resistència de partides armades o maquis, persecució de la llengua i la cultura catalanes, fèrria censura, abolició de les institucions i destrucció de les senyes d'identitat. A pesar de tant odi i acarnissament, impressiona com la societat civil catalana es va refer econòmicament i va mantenir la pervivència del sentiment nacional. En ple debat de l'Estatut, la lectura del llibre de Torras és recomanable per a catalans i per a espanyols." ${ }^{60}$ 
Au-delà de la destruction programmée de l'État catalan, l'Espagne a également organisé le démantèlement, ou l'écartèlement pourrait-on dire, de la nation catalane, en collaboration avec les autres puissances hégémoniques européennes lors de la signature du Traité d'Utrecht en 1713. Cette thèse n'apparait que chez les tenants de l'hégémonie catalane dans les territoires appelés « Pays Catalans », c'est-à-dire tous les territoires de langue catalane ou plus généralement chez les tenants de l'hégémonie de la Catalogne sur l'ensemble de la Couronne d'Aragon. La nation catalane aurait ainsi été écartelée entre la France (la Catalogne du Nord), l'Italie (Alguer en Sardaigne), l'Espagne (Valence, Catalogne et Majorque) et l'Angleterre, du moins jusqu'en 1802 (Minorque). La nouvelle histoire se propose donc comme objectif non seulement de contraindre les Castillans à réécrire l'historiographie d'État, mais également toutes les puissances hégémoniques de l'époque, à savoir la France, l'Angleterre et l'Italie, quand ce n'est pas l'historiographie mondiale :

El primer que farà Catalunya, si mai és un Estat independent, serà indubtablement, reescriure la història en la seva integritat a partir de la veritat dels fets [...]. I l'Estat espanyol haurà de encarar, amb totes les conseqüències, l'evidència d'una manipulació que impactarà [...] en el conjunt d'històries estatals d'Europa i de mig món. ${ }^{61}$

21 La méthode consiste à susciter chez le lecteur un sentiment d'indignation en révélant, par l'intermédiaire d'un montage de listes et de citations décontextualisées, la cruauté et l'iniquité des Castillans et des Bourbons. L'un des objectifs visés est de permettre de faire des rapprochements, des parallélismes avec d'autres époques, notamment la décolonisation africaine, l'occupation de l'Europe par les Nazis et la Guerre Civile :

El que resulta més esfereïdor en aquest contundent aplec documental és la combinació de dos elements: l'absolut menyspreu que mostren aquests papers respecte al nostre país i la seva gent, sovint arribant a l'odi, i la naturalitat amb que els conqueridors castellans i els botiflers dissenyen i apliquen unes mesures que, en definitiva, són les línies d'una política d'extorsió i de terror que són un clar antecedent del que faran els nazis a Alemanya (1933-1945) i a l'Europa ocupada (1939-1945) i els espanyols feixistes del general Franco entre 1936 i $1975 . .{ }^{62}$

Le processus d'indépendance devient donc processus de libération nationale, lequel se révèle être l'unique solution envisageable pour non seulement récupérer la souveraineté mais aussi pour recomposer le corps et le cœur de la nation catalane en délivrant le peuple élu de l'esclavage de Pharaon. On est là face à ce que l'on pourrait appeler un "complexe de la sortie d'Égypte", dont le propos est d'engager ce que beaucoup voient comme un processus de décolonisation.

\section{Conclusion}

Il est difficile d'évaluer l'impact de ces théories sur la population et des études de terrain seraient utiles à une meilleure compréhension de ce phénomène. On pourra, malgré tout, tenter d'avancer quelques hypothèses. Premièrement, ce discours pseudohistorique s'articule autour d'une dualité entre "pactisme» et "absolutisme». La capitulation de Barcelone le 11 septembre 1714 face aux troupes de Philippe V marque une rupture dans le processus de construction démocratique de la Catalogne, voire de l'Espagne pour certains. En abolissant les libertés catalanes et l'institution des Cortès, le décret de Nueva Planta de 1716 anéantit simultanément la tradition parlementaire qui s'était transmise depuis le Moyen Âge. L'éditorial "Repressió a la Generalitat" précise 
en effet que la Catalogne peut se targuer d'avoir eu des "Institucions que un dia van produir les constitucions més avançades de la seva època segons molts historiadors europeus." ${ }^{3}$ Aussi l'indépendantisme deviendrait-il un moyen de renouer avec cette tradition démocratique, une thèse qui, sans doute, a davantage de force que celle de la supposée existence d'un État catalan qui aurait été annihilé en 1714. Dans l'historiographie catalane, y compris chez les historiens professionnels, le "pactisme " catalan s'oppose à l'absolutisme et devient un modèle démocratique de droit constitutionnel qui serait aux origines de la revendication actuelle d'une démocratie participative. Les Cortès étaient en effet des assemblées réunies par le roi auxquelles siégeaient des représentants des trois ordres de la société catalane. Elles ne sont certes pas présentées comme des modèles de démocratie avancée par tous, mais plutôt comme l'ancêtre de la démocratie parlementaire, laquelle est appelée à évoluer vers la démocratie participative. On est là face à ce que l'on pourrait appeler un «darwinisme de la démocratie », une évolution des pratiques démocratiques que les Castillans, par essence dictatoriaux, auraient interrompue brutalement en 1714.

Deuxièmement, l'indépendantisme historicisé devient un moyen de protestation contre les politiques de l'État espagnol et contre le centralisme prôné par Mariano Rajoy. La réécriture de l'histoire semble fonctionner comme un catalyseur de revendications politiques et la proclamation de l'indépendance de l'État catalan permet de faire converger autour d'un projet fantasmé, présenté sous la forme d'une utopie, les frustrations de classes sociales très hétérogènes, voire irréconciliables. L'histoire, en tant que discipline, passe donc d'un statut contemplatif - celui du chercheur - à un statut actif et militant. Peu importe que le discours tenu soit vrai ou faux : l'objectif est d'agir, ou plutôt de "faire ", comme on l'entend dans l'expression très répandue de "fer país"64. Dit autrement, l'histoire n'est plus conçue comme une explication des causes ou comme la description de phénomènes passés mais davantage comme un levier d'action politique. Ces deux sites et plus largement l'idée selon laquelle la Catalogne est une colonie de l'Espagne émergent et se consolident dans un contexte politique, social et économique bien défini. À l'origine, il y a certes le recours en inconstitutionnalité engagé par des députés du Patrido Popular auprès du Tribunal Constitutionnel au sujet du nouveau statut d'autonomie catalan de 2006. Celui-ci avait été, rappelons-le, approuvé par le parlement catalan (120 voix pour et 15 voix contre) puis par un référendum, où le « oui » l'avait emporté à plus de 70\%. La publication de la sentence en 2010, par laquelle le Tribunal Constitutionnel déclarait anticonstitutionnels certains articles, a été interprétée à la fois comme une attaque contre la souveraineté populaire mais aussi comme une rupture définitive de la coexistence pacifique entre la Catalogne et la Castille. L'historien contemporanéiste de l'Université de Barcelone, Antoni Segura, a consacré en 2013 un essai dans lequel il retrace la généalogie de l'indépendantisme. Il affirme que "per a gran part de ciutadans de Catalunya aquella sentència tancava definitivamente la via estatutària $i$, de retruc, negava qualsevol possibilitat d'encaix satisfactori de Catalunya amb Espanya"65. Et d'ajouter que les manifestations organisées en Catalogne en réaction contre la sentence du Tribunal Constitutionnel avaient précipité la "ruptura del darrer punt de connexió : el sentimental" ${ }^{66}$. Aussi y a-t-il urgence à libérer la société catalane du joug de son oppresseur, de rompre les chaînes qui la lient depuis plusieurs siècles à la métropole et à fonder un nouvel ordre, une nouvelle sociétée ${ }^{67}$. Selon Jaume Sobrequés, "la història ensenya que els processos colonitzadors només han estat vençuts quan els països víctimes de l'acció destructiva de la metròpoli han assolit la independència". Or, ajoute- 
t-il, la Catalogne est l'un des rares pays de la planète à ne s'être pas encore libéré de l'oppression du colonisateur, avant de conclure que "continua essent [...] l'única colònia que encara existeix al vell continent" ${ }^{68}$. Dans le système idéologique que proposent la pseudo-histoire, le "pactisme » catalan constitue un modèle de démocratie et agit comme aspiration à la liberté que l'Espagne, incarnée par le gouvernement de Mariano Rajoy, s'attache méticuleusement à étouffer. Le processus souverainiste apparaît par conséquent comme un mouvement de libération nationale ou, serait-on tenté de dire, un mouvement national de libération.

Mais il pourrait y avoir aussi une explication d'ordre plus sociale, voire sociologique qui justifierait la poursuite de l'enquête. En effet, la lecture de ces articles montre bien que si le discours autour de la préservation de la langue catalane occupe une place importante, celui qui se constitue autour de la critique envers l'État est tout aussi fort, sinon plus. Le discours indépendantiste serait-il, avant tout, un catalyseur d'insatisfaction, notamment pour ce qui relève de la politique économique depuis les mesures d'austérité mises en place par le gouvernement de José Luis Zapatero et renforcées par ceux de Mariano Rajoy? Le discours pseudo-historique se configure certes comme une réaction contre la censure de la langue, mais aussi contre la spoliation économique et la politique jugée autoritaire et centralisatrice du gouvernement castillan depuis l'époque moderne. Or, des enquêtes réalisées à la suite des élections au parlement catalan de décembre 2017 montrent que l'électorat indépendantiste appartient à la classe moyenne supérieure ${ }^{69}$. L'électeur indépendantiste type dispose d'un revenu mensuel situé entre 2400 et $3000 €$. Il est né en Catalogne, de parents et de grands-parents nés en Catalogne et il est employé dans le secteur privé en contrat indéterminé ou retraité. Il est marié, blanc, parent d'un ou deux enfants et catholique non pratiquant ou athée (la proportion de personnes se déclarant catholiques non pratiquant est supérieure à celle se déclarant athée). Il vit dans l'intérieur de la Catalogne, soit en zone rurale soit à Gérone ou Lérida. Son idéologie repose sur trois grands pôles: la préservation de la langue catalane, le désaccord envers le système de subsidiarité et une défiance envers les institutions d'État et les politiques professionnels. Loin de répondre à une conviction profonde, la proclamation de l'indépendance est conçue comme la seule issue à l'immobilisme de l'État, à la corruption de la classe politique et comme une réaction d'indignation face à l'application de l'article 155, à la suite du référendum du $1^{\mathrm{er}}$ octobre 2017. Le nationalisme catalan actuel et sa mutation vers l'indépendantisme ne sont-ils pas le résultat d'une atomisation et d'un déclassement - ou d'un sentiment de déclassement de la classe moyenne supérieure catalane à la suite de la crise économique des années 2000 ?

\section{NOTES}

1. Propos de Patrick Boucheron dans l'émission Drôle d'endroit pour une rencontre, France 5, disponible sur https://www.youtube.com/watch?v=SBPUce3xuV0, consulté le 19 septembre 2018. 2. Fernando Savater, Figuraciones mías, Barcelona, Ariel, 2013, p. 133. 
3. Entre la tenue du colloque en novembre 2018 et la publication de l'article, un collectif d'historiens catalans et valenciens ont publié un ouvrage consacré à la pseudo-histoire. Vicent Baydal et Cristian Palomo, Pseudohistòria contra Catalunya. De l'espanyolisme a la Nova Història, Vic, Eumo Editorial, 2020. Le livre propose une approche plus large et plus approfondie que ne le fait cet article, mais les conclusions auxquelles nous parvenons sont proches. Nous proposons une recension de l'ouvrage en fin de numéro.

4. Jordi Bilbeny «Qui som? El patronat », https://web.archive.org/web/20140713163145/http:// www.inh.cat/patronat, consulté le 3 août 2018.

5. «Qui som », https://www.inh.cat/institut, consulté le 6 août 2018.

6. « Qui som?», http://www.cch.cat/php/ls.php?fx=1mtqui, consulté le 3 août 2018.

7. Ibid.

8. Àngel Casals Martínez, "La quimera substituint la Història", El País, 26 novembre 2014.

9. Jordi Bilbeny, "Sobre l'origen de la bandera dels Estats Units d'Amèrica ": "Llavors, ara que sabem per les meves recerques, i pels matisos que hi han afegit un gran tou d'investigadors en àmbits molt diversos, que el nou continent va ser descobert, conquerit, evangelitzat i poblat per catalans i que els escuts i les banderes catalanes apareixen en multitud de planifesferis, mapamundis i cartes de navegació; en múltiples gravats, pintures, llibres, banderes (tant de naus com d'exèrcits), ara és més fàcil interpretar-ho: la bandera dels EUA només i únicament té la catalana com a rerefons. Barres vermelles idèntiques", sur https://www.inh.cat/articles/Sobrel'origen-de-la-bandera-dels-Estats-Units-d'America, consulté le 6 août 2018.

10. Xosé Carlos Caneiro, "Independentismo y dignidad”, La Voz de Galicia, 14 novembre 2017.

11. Henry Kamen, España y Cataluña. Historia de una pasión, Madrid, La esfera de los libros, 2014, p. 10.

12. Henry Kamen, “No hay ningún expolio, Cataluña es la región más rica y eso también es 'culpa' de España”, Abc, 6 septembre 2014.

13. Henry Kamen, España y Cataluña, op. cit., p. 10.

14. "Qui som", https://www.inh.cat/institut, consulté le 6 août 2018.

15. Edmond Cros, «La notion d'idéosème », https://www.sociocritique.fr/?La-notion-dideoseme, consulté le 3 septembre 2018.

16. Interview de Jordi Bilbeny, "Jordi Bilbeny contra la manipulació de la història", https:// www.inh.cat/articles/Jordi-Bilbeny-contra-la-manipulacio-de-la-historia, consulté le 9 juillet 2018.

17. David Morgades, "La importància de ser Jordi Bilbeny", 14 octobre 2011, INH, https:// www.inh.cat/articles/La-importancia-de-ser-Jordi-Bilbeny, consulté le 4 septembre 2018.

18. La thèse de la catalanité de Christophe Colomb n'est pas nouvelle et Jordi Bilbeny la reprend, en s'en attribuant l'autorité, à Luis Ulloa qui la développe dans son ouvrage intitulé Xristoferens Colom y Fernando el Católico, Paris, Maisonneuve frères, 1928. Voir le compte rendu que lui consacre Raoul d'Harcourt, «Christophe Colomb catalan », Journal de la société des Américanistes, 1930, no22-1, p. 231.

19. Interview de Jordi Bilbeny, « Jordi Bilbeny contra la manipulació de la història », op. cit.

20. «Entrevista a Jordi Bilbeny, cap de recerca de l'Institut Nova Història », https://www.inh.cat/ articles/Entrevista-a-Jordi-Bilbeny,-Cap-de-recerca-de-l'Institut-Nova-Historia.-Diari-de-Prada, consulté le 11 juillet 2018.

21. "La confirmació de la sospita", Cercle Català d'Història, http://www.cch.cat/php/ls.php? $\mathrm{fx}=1 \mathrm{z} 09030100$, consulté le 11 juillet 2018.

22. Ibid.

23. Andreu Marfull, “Censura o judici”, https://www.inh.cat/articles/Censura-o-Judici, consulté 11 juillet 2018.

24. Joan Calsapeu, "Sobre Inquisició i Decadència", 16 mai 2018, https://www.inh.cat/articles/ Sobre-Inquisicio-i-Decadencia-, consulté le 4 septembre 2018. 
25. Biel Ferrer Puig, “Contra la Decadència, un bon alumne”, $1^{\text {er }}$ août 2018, https://www.inh.cat/ articles/Contra-la-Decadencia,-un-bon-alumne\#comments consulté le 5 septembre 2018.

26. Jordi Bilbeny, Inquisició i Decadència, Barcelone, Librooks, 2018, "Prefaci», p. XVIII. Remarquons que l'édition de ce livre a été rendue possible grâce à une campagne de financement lancée par l'Institut Nova Història (crowdfounding). Cette campagne, qui entendait récolter $3500 € \mathrm{a}$, en fin de compte, récolté $7110 €$ près de 200 jours avant sa fermeture. Voir https:// www.inh.cat/micromecenatge/inquisicio-i-decadencia, consulté le 5 septembre 2018. La couverture du livre présente un dessin de la tête de Cervantes traversé, au niveau de la bouche, d'une bande rouge, rappelant l'imagerie lancée par l'ANC lors de la votation d'octobre 2017, symbole de la répression exercée par l'État espagnol sur la liberté d'expression des Catalans.

27. Lluís Maria Mandado, El Quixote va esborrar el Quixot, Barcelone, Llibres de l'Index, 2013. La maison d'édition des Llibres de l'Índex: "Llibres de l'Índex pren el seu nom de l"Index Librorum Prohibitorum', el llistat de llibres que, des del papat de Pius V, l'any 1559, fins a Pius XII, l'any 1948, l'Església catòlica prohibia allà on tenia poder politicosocial. En aquesta llista, hi van ser tots els llibres bàsics de la ciència des de Galileu a Newton o de filòsofs i escriptors contemporanis com ara Sartre i Moravia. El dimoni del logotip és obra de la dissenyadora barcelonina Pati Nuñez", http://llibresindex.blogspot.com/, consulté le 6 septembre 2018.

28. Martín de Riquer, Cervantes en Barcelona, Barcelone, Cuadernos del Alcantilado, 2005, p. 9-17.

29. Interview de Jordi Bilbeny par Jordi Vilarrodà, le 30 janvier 2013, https://www.inh.cat/ articles/'El-Quixot-es-la-traduccio-d-una-obra-catalana,-d-en-Joan-Miquel-Servent, consulté le 5 septembre 2018.

30. Antoni Balbuena Tusell, La vida de Llatzer de Tormos, Barcelone, Llibres de l'Índex, 2013.

31. On pourra retrouver l'ensemble des détails de l'argumentation dans une série de trois articles que Jordi Bilbeny a publiés sur son blog personnel: http://www.jordibilbeny.cat/2016/10/24/ mites-i-llegendes/, http://www.jordibilbeny.cat/2017/04/20/el-lazarillo-de-tormes-va-serescrit-en-catala-iiiii/ et http://www.jordibilbeny.cat/2017/04/20/el-lazarillo-de-tormes-va-serescrit-en-catala-iiiiii/, consultés le 6 septembre 2018.

32. "La vida de Llàtzer de Tormos. Estudi preliminar de Jordi Bilbeny", https://www.inh.cat/ articles/La-vida-de-Llatzer-de-Tormos.-Estudi-preliminar-de-Jordi-Bilbeny, consulté le 6 septembre 2018.

33. Àngel Casals lors d'une conférence organisée par le $\mathrm{CCH}$ sur le thème suivant: "El paper de la història en el camí cap a la soberania" (http://www.cch.cat/php/ls.php? $f_{x}=1 m e \_d x \& p a r a u l e s=J o s e p \% 20 C a t \% E 0 \&$ document=*ART, consulté le 6 septembre 2018). Précisons ici que le $\mathrm{CCH}$ est beaucoup plus ambigu que l'INH. Si ce dernier a, dès ses origines, fait de la pseudo-histoire et a très vite été critiqué par les historiens de métier, le $\mathrm{CCH}$ a bénéficié de la participation d'historiens et d'historiennes reconnus, à l'instar d'Àngel Casals et Eva Serra, dont l'intégrité scientifique n'est nullement à remettre en cause et qui semblent avoir progressivement pris leurs distances. Une étude plus approfondie, notamment de la chronologie des événements, pourrait montrer une inflexion dans l'évolution du $\mathrm{CCH}$ qui, à bien des égards, correspond dans ses premiers temps du moins à ce que nous connaissons en France comme les Universités populaires. Nous le posons du moins ici sous la forme d'une hypothèse.

34. Andreu Marfull, “Censura o judici”, le 23 janvier 2015, https://www.inh.cat/articles/Censurao-Judici, consulté le 6 septembre 2018.

35. "La confirmació de la sospita", op. cit.

36. Éditorial "Neix el Cercle Català d'Història", http://www.cch.cat/php/ls.php?fx=1z08120100, consulté le 21 septembre 2018.

37. Eva Sans, «La perscució de Catalunya », 21.1.2011, http://www.cch.cat/pdf/ persecusio_de_catalunya.pdf, consulté le 19.9.2018.

38. Lluís Batlle i Rossel, Sota l'estora del Siglo d'or castellà. Empremtes catalanes als segles XVI i XVII, Barcelone, Quixot.cat, 2017, p. 9-11. 
39. Joaquim Ullan Martorell, «La manipulación documental sobre los hechos del descubrimiento de América: la exclusión de la corona Catalano-aragonesa ». Communication présentée à l'occasion du 54 Congreso Internacional de Americanistas qui s'est tenu à Vienne entre les 15 et 20 juillet 2012. Le texte est disponible sur le site du $\mathrm{CCH}$, http://www.cch.cat/pdf/ 54ica_ponencia_jullan.pdf, consulté le 19.9.2018.

40. Idem. Il serait intéressant d'étudier l'éventuelle relation entre le développement de cette « nouvelle histoire » et le boom éditorial des publications sur le développement personnel.

41. Jordi Bilbeny, « 12 d'octubre, robatori i espoliació sense límits », op. cit.

42. Joaquim Ullan Martorell, "Métode historiogràfic o policial? Refexions d'una espoliació historiogràfica”, 3 décembre 2009, http://www.cch.cat/pdf/reflexions_01.pdf, consulté le 20 septembre 2018.

43. Joaquim Ullan Martorell, "La manipulación documental sobre los hechos del descubrimiento de América: la exclusión de la corona Catalano-aragonesa”, op. cit.

44. Andreu Mas, "La història manllevada per fer-nos petits", INH, 23 mars 2012, https:// www.inh.cat/articles/La-historia-manllevada-per-fer-nos-petits, consulté le 21 septembre 2018. El Bulli est le restaurant de Ferran Adrià.

45. Carlo Ginzburg, Il Formaggio e i vermi. Il cosmo di un mugnaio del Cinquecento, Turin, Einaudi, 1976. Traduit en français par Monique Aymard, Le Fromage et les vers. L'univers d'un meunier du $\mathrm{XVI}^{\mathrm{e}}$ siècle, Paris, Aubier, 1980.

46. Arthur Conan Doyle, Le Signe des Quatre [1890], Paris, Le Livre de Poche, 1995. Voir le chapitre I : « La déduction est une science ».

47. Joaquim Ullan Martorell, "Cròniques des de la Sevilla Colombina”, http://www.cch.cat/pdf/ sevilla.pdf, consulté le 19 septembre 2018.

48. Joaquim Ullan Martorell, "Métode historiogràfic o policial? Refexions d'una espoliació historiogràfica", op. cit.

49. Ibid.

50. Antoni Muñoz i Josep Catà, Ambaixadors catalans a Madrid. Els inicis de la Guerra de Separació (1640-1641), Barcelone, Rafael Dalmau Editor, 2015. Voir Mathias Ledroit, « La Révolte des Catalans (1640) au ralenti », dans l'Âge d'or, 끈. 10.

51. Paul Ricœur, La mémoire, l'histoire, l'oubli, Paris, Édition du Seuil, 2000, p. 96-105.

52. Mathias Ledroit, "Les ambassadeurs catalans à Madrid en 1640: chronique d'une guerre annoncée? ", dans Béatrice Perez (dir.), Ambassadeurs, apprentis espions et maîtres comploteurs. Les systèmes de renseignement en Espagne à l'époque moderne, Paris, PUPS, 2010, p. 333-341.

53. Jaume Sobrequés i Callicó, “Catalunya és una colònia d'Espanya”, El Punt/Avui, 11 novembre 2015.

54. Marc Pons, "Què va arrasar el Decret de Nova Planta", El Nacional.cat, 29 octombre 2017.

55. Ibid.

56. Jaume Sobrequés i Callicó, "Catalunya és una colònia d'Espanya”, op. cit.

57. Jordi Bilbeny, "Sobre la història, la Guerra de Successió i l'abducció premeditada de la nostra història", https://www.inh.cat/articles/Sobre-la-historia,-la-guerra-de-successio-i-l-abducciopremeditada-de-la-nostra-consciencia, consulté le 4 octobre 2019.

58. Andreu Mayoyo i Artal, "Borbó a mata-degolla", El Periódico, dans le supplément Llibres, 8 septembre 2005, p. VII.

59. Jaume Sobrequés i Callicó, “Catalunya és una colònia d'Espanya”, op. cit.

60. Andreu Mayoyo i Artal, "Borbó a mata-degolla", op. cit.

61. Andreu Marfull Pujadas, "La catalanofòbia: el fenomen, les causes, les conseqüències i la solució”, https://www.inh.cat/articles/La-catalanofobia-el-fenomen,-les-causes,-lesconsequencies-i-la-solucio, consulté le 5 octobre 2018. 
62. Recension par Jordi Peñarroja du livre d'Antoni Muñoz et Josep Catà, Resistència borbònica $i$ resistència catalana (1714-1736), paru aux éditions Rafael Dalmau, 2005, http://www.cch.cat/php/ ls.php? $\mathrm{fx}=1 \mathrm{z} 08120200$, consulté le 4 octobre 2018.

63. "Repressió a la Generalitat", op. cit.

64. Voir, entre autres, à ce propos les spots publicitaires de la Diputació de Barcelona sur leur chaîne youtube: https://www.youtube.com/watch?v=t8xzf7YcT3w, consulté le 11 octobre 2018.

65. Antoni Segura, Crònica del catalanisme. De l'autonomia a la independència, Barcelone, Angle Editorial, 2013, p. 31.

66. Ibid., p. 32.

67. Antoni Muñoz, Josep Catà et Jordi Peñarroja, "El paper de la història en el camí cap a la soberania", http://www.cch.cat/pdf/taula_11s_a.pdf, consulté le 4 octobre 2018.

68. Jaume Sobrequés i Callicó, “Catalunya és una colònia d'Espanya”, op. cit.

69. Enquesta sobre context polític a Catalunya 2018, REO, $n^{\circ} 874,23$ février 2018, http:// upceo.ceo.gencat.cat/wsceop/6508/Taules\%20estad\%C3\%ADstiques\%20-874.pdf, consulté le 17 octobre 2018.

\section{RÉSUMÉS}

La « Nova Història », la « nouvelle histoire », est un courant de pseudo-histoire qui apparaît dans l'espace public en 2007. Elle se propose de révéler la spoliation et l'oppression que l'Espagne exerce sur la Catalogne depuis 1469. Cette réécriture du passé entend diffuser un sentiment d'angoisse et d'urgence dans la société catalane, urgence de proclamer l'indépendance pour rompre les chaînes de la colonisation avec lesquelles l'État espagnol a entravé les Catalans. Il ne s'agira pas, dans cet article, de rétablir les faits ni de s'interroger sur la pertinence de ce courant. On envisagera cette "nouvelle histoire " comme un système doté d'une cohérence interne et dont on proposera un premier essai de synthèse.

La "Nova historia", la "nueva historia" es una corriente de pseudo-historia que brotó en el espacio público en 2007. Se dedica a revelar la espoliación y la opresión que ejerce el Estado español sobre Cataluña desde 1469. Esta reescritura del pasado pretende sembrar un sentimiento de zozobra y urgencia en la sociedad catalana, para animar a los ciudadanos a proclamar la independencia. No se tratará en este artículo de restablecer la verdad del hecho histórico ni tampoco de interrogarse sobre la pertinencia de esta corriente. Al contrario, consideraremos esta "nueva historia" como un sistema dotado de una coherencia interna y propondremos un primer intento de síntesis.

\section{INDEX}

Mots-clés : Nova història, urgence, pseudo-histoire, Catalogne, indépendantisme

Palabras claves : Nova història, urgencia, pseudo-historia, Cataluña, independentismo 
AUTEUR

MATHIAS LEDROIT

Laboratoire LISAA, Université Gustave-Eiffel 